\title{
Non-Iterative Model Based Image Reconstruction of Diffuse and Radiative Transfer Optical Tomography in Quality Control on Agricultural Produce Studies
}

\author{
Vebi Nadhira $^{1 *}$, E. Juliastuti ${ }^{1}$, Agah D. Garnadi ${ }^{2}$, Deddy Kurniadi ${ }^{1}$, and Yoko Hoshi ${ }^{3}$ \\ ${ }^{1}$ Instrumentation and Control Research Group, Faculty of Industrial Technology \\ Institute Technology of Bandung Ganesha 10,+62 22250 4424, 40132 Bandung Indonesia \\ ${ }^{2}$ Departement of Mathematics, Faculty of Mathematics and Natural Sciences \\ Bogor Agricultural University, J1 Meranti, Kampus Darmaga, 16680, Bogor, Indonesia \\ ${ }^{3}$ Integrated Neuroscience Research Project, Tokyo Metropolitan Institute of Medical Science \\ 2-1-6 Kami-kitazawa, Setagaya, Tokyo 156-8506, Japan \\ Email : vebi@tf.itb.ac.id
}

\begin{abstract}
In the previous study, we developed the non-iterative image reconstruction based on diffusionequation.Within this research, we applied the same non-iterative algorithm scheme using radiative transfer equation. Basically, the non-iterative image reconstruction was a development of model based image reconstruction that implemented truncated singular value decomposition and L-curve analysis to solve the ill-posed problem.These algorithm reduces the computation time to reconstruct the cross sectional area.As part of the continuing development of agricultural produce quality control based on optical tomography, potato experiment was conducted to evaluate these two non-iterative algorithms. The object was illuminated by the near infrared source from 8 positions on object's boundary.In this experiment, we vary the position and amount of epoxy as targets on the object then we analyze the residual value between measurement and reconstructed boundary data. The reconstructions were performed with continuous-wave domain.Furthermore, we compare the residual value fromdiffuse optical tomography and radiative transfer optical tomography. The result of this study indicated that these algorithmshave shown promising to detect the presence of epoxy on potato which is significant for agricultural produce quality control.
\end{abstract}

Keywords:truncated singular value decomposition, L-curve, residual value, potato.

\section{INTRODUCTION}

Several researchers have been developing the reconstruction methods to reduce the computation time in optical tomography (OT) with the regularization parameter selection method [7], a fast forward solver of radiative transfer equation (RTE) [5], non-iterative algorithm [8], hardware development [14], and signal processing approach [1, 13, 15].In this paper, we focused on study of the non-iterative image reconstruction based on diffusion equation (DE) and RTE.To avoid the iterative step, we used L-curve-based algorithm for selection $k$ optimal automatically to obtain truncated singular value decomposition (TSVD) matrix. This matrix will derive a new well-posed problem. In forward solving, the continuous wave signal processing approach was applied in order to get a faster boundary data calculation. Furthermore, we applied a fast RTE solver that has been developed by $\mathrm{H}$. Gao and $\mathrm{H}$. Zhaoto solve the forward problem in radiative transfer optical tomography (RTOT).

Usually, the development of non-destructive evaluation (NDE) based on optical tomography (OT) method has been focused for medical application [4, 6, 8, 9]. Nevertheless, NDE OT development on other application is also interesting and promising part as described by some researcher on this field [3, 14]. This paper as well as our previous papers mainly study NDE based on OT method for agricultural product quality control [17, 18, 19]. Therefore, the experiment of internal defects monitoring on agricultural produce using OT is described on this paper to validate our proposed non-iterative image reconstruction algorithm.

The rest of the paper is organized as follows. The principle of OT, forward problem using DE and RTE, finite element method (FEM) theory, non-iterative model based image reconstruction (NIMoBIR) theory, TSVD and L-curve analysis are reviewed in section 2.Section 3 describes experiment set up. Simulation results are shown in section 4. Finally, the conclusions are given in section 5.And last section is for the list of references.

\section{NIMOBIR OT}

\section{A. The principle of $O T$}

OT reconstruction system is divided into two essential parts. The first one is forward problem solving and the other one is inverse problem solving [13, 21], as shown in Figure 1.The forward problem solving is used to calculate the boundary data of prediction model and/or simulation object when the optical properties of the medium and the amount of input light are given.In this paper, we used DE and RTE to describe the light propagation in biological material, which then are used as the forward problem solving [1, 5, 16].While inverse problem solving is an algorithm to predict an optical 
coefficient distribution inside the medium by compare the boundary data from "object" and prediction model.

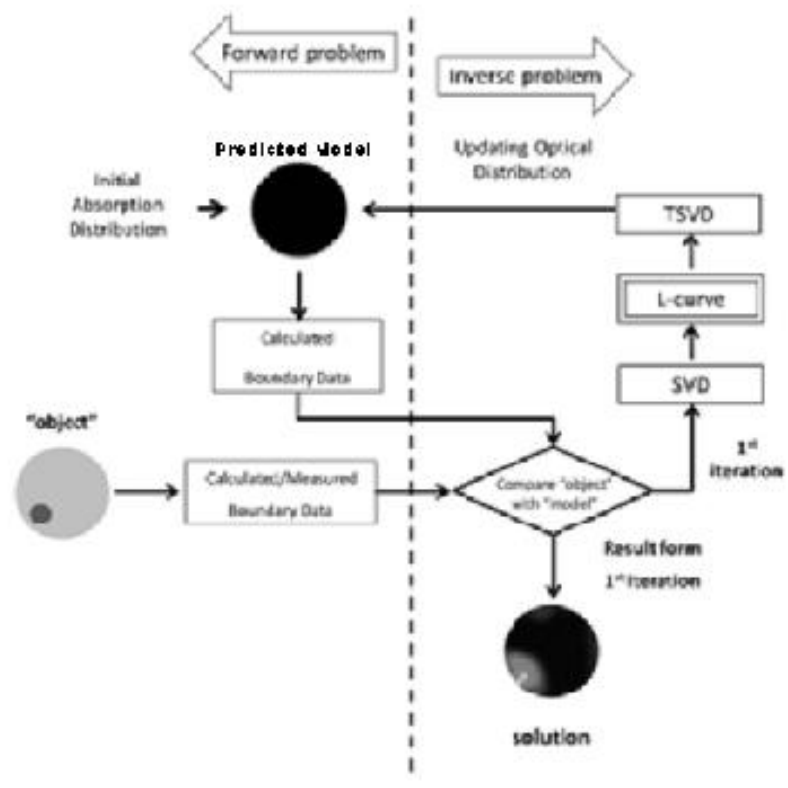

Fig. 1. NIMoBIR algorithm[21]

A fundamental model for light transport in tissues is the radiative transfer equation. The continuous wave domain RTE is of the form

$$
\hat{s} . \nabla . L(r, \hat{s})+\left(\mu_{a}+\mu_{s}\right) L(r, \hat{s})=\mu_{s} \int_{s^{n-1}} f\left(\hat{s}, \hat{s}^{\prime}\right) L(r, \hat{s}) d \hat{s}^{\prime}+q(r, \hat{s})
$$

where $\mu a$ and $\mu$ sare absorption and scattering coefficient of the medium, respectively. Furthermore, $L(r, \hat{s})$ is the radiance, $f\left(\hat{s} . \hat{s}^{\prime}\right)$ is the probability density function. The probability density function describes the probability of scattering from direction $\hat{s}$ into direction $\hat{s}^{\prime}$ due to scattering event. $q(r, \hat{s})$ is the source per unit volume at position $\mathrm{r}$ along $\hat{s}$.Even though, RTE is a widely accepted model, but DE is more commonly used because of its computational simplicity. In the DE framework, the RTE is approximated as the following

$-\kappa \nabla^{2} . \Phi(r)+\mu_{a} \Phi(r)=q_{0}(r)$

where $\Phi$ is the diffuse photon fluence rate, $q_{0}$ isthe integrated light source term, and $\kappa$ is the optical diffusion coefficient [1, $2,15]$. The optical diffusion coefficient can be represented as follows:

$$
\kappa=\frac{1}{3\left[\mu_{s}^{\prime}+\mu_{a}\right]}
$$

where $\mu_{s}{ }^{\prime}\left[\mathrm{m}^{-1}\right]$ the reduced scattering coefficient $[13,16]$. The optical tomography system that use DE is usualy called diffuse optical tomography (DOT).
In order to simplify the boundary data calculation process using forward problem, we need to apply discretization approach. In this paper FEM approach is used to divided the object or model into non-overlapping triangle elements as shown in Figure 2.

Furthermore, to simplify the computational process, FEM approach is applied to simulation object and prediction model. This FEM approach is dividing the object or model into non-overlapping triangle elements in other to discretize the diffusion equation as shown in Figure 2.

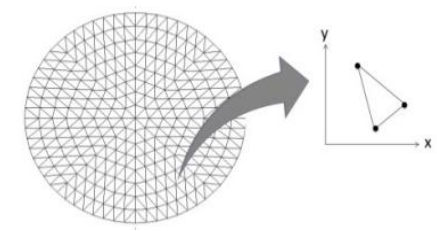

Fig. 2. Discretization object with FEM[21]

FEM is selected because it can provide fast, accurate and flexible modeling for complex geometries [17, 19]. By applying FEMto DE and RTE, the photon density $\Phi$ and the radiance $L$ is approximated by a piecewise linear and continuous function, $\Phi^{\mathrm{h}}$ and $L^{\mathrm{h}}$, respectively, as follows:

$\Phi^{h}(r)=\sum_{j=1}^{N} \Phi_{j}(r) u_{j}(r)$

$$
L^{h}(r)=\sum_{j=1}^{N} L_{j}(r) u_{j}(r)
$$

where $u_{j}$ arelinear basis functionsfor which the values at element centres $n_{j}$ are defined as $u_{i}\left(n_{j}\right)=\delta i j(i, j=1, \ldots, N)$. This $\Phi^{\mathrm{h}}$ or $L^{\mathrm{h}}$ will be used as input for inverse problem.

In the image reconstruction algorithm of OT, the optical coefficient distribution of the "object" or/and predictive model are estimated based on boundary data and light transport propagation within the medium.

The boundary data from both DE and RTE can be described as a general vector $b$ and the optical coefficient distribution can be described by a general vector $\mu$. Then, a physical model that describes the relation between $b$ and $\mu$ can be written as the following

$$
J=\frac{\partial b}{\partial \mu}
$$

where $J$ is the sensitivity matrix that defines sensitivity of $F$ to perturbations in $\mu$, also known as Jacobian matrix. Furthermore, the forward problem is defined as to find the values of data $b$ fromgiven $\mu$, for which we have

$b=J \mu$ 
Whilein the inverse problem, we have to estimate the image which represent optical coefficient distribution $\mu$ from given data $b$. We may then write

$$
\mu=J^{-1} b
$$

Unfortunately, the Jacobian inversion process leads to illposed problem. Generally, this problem can be solved by applying iterative reconstruction. But, we applied TSVD to solve this problem in a non-iterative way. The first step in TSVD algorithm is to convert matrix $J^{-1}$ to singular value decomposition matrix as follows:

$$
J^{-1}=V S D^{T}
$$

where. $\mathrm{S}=\operatorname{diag}\left(s_{1}, \ldots, s_{\mathrm{n}}\right)$ has non-negative diagonal elements appearing in non-increasing order [12]. In this case, $V=\left(v_{1}, \ldots, v_{\mathrm{n}}\right)$ and $D=\left(d_{1}, \ldots, d_{\mathrm{n}}\right)$ are matrices with orthonormal columns. Furthermore, to derive a new problem with well-posed rank deficient coefficient matrix, we need to truncate $S$ matrix.

The rank deficient matrix, $S_{\mathrm{k}}$, is obtained by truncating the $\mathrm{S}$ at k so that $S_{\mathrm{k}}$ isthe best rank- $k$ approximation to $S . S_{k}$ is given by

$$
S_{k}=\sum_{i=1}^{k} s_{i}, \quad k \leq n .
$$

To determine $k$ optimal, L-curve analysis is the most convenient graphical tool for choosing $k$ optimal precisely [11]. L-curve is plottingthe norm $\|\mu\|_{2}$ of the SVD solution tothepredictionerrornorm $\left\|S_{k} \mu-b\right\|_{2}$, where $k$ optimal minimizes these two quantities at the same time.

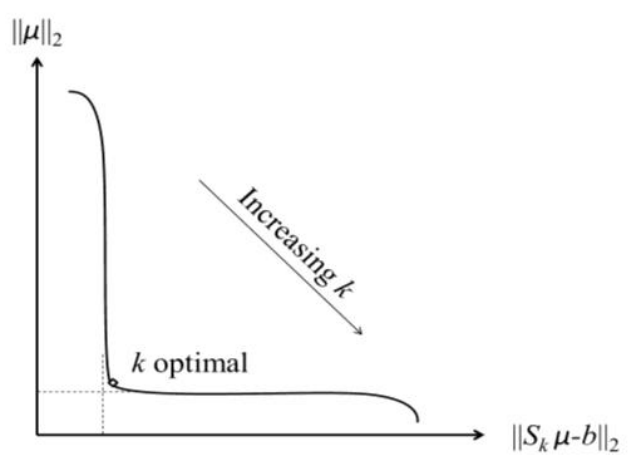

Fig. 3. L-curve analysis for TSVD [20]

\section{EXPERIMENT OF POTATOMONITORING}

To evaluateour non-iterative model based image reconstruction method on a ring array diffuse optical tomography system for quality control on potato, we perform measurement using cylindrical carrot with the diameter and height was $30 * 10^{-3} \mathrm{~m}$ and $40^{*} 10^{-3} \mathrm{~m}$, respectively.
Theexperiment was done on four different circular objects with several defect conditions as shown in Figure5.

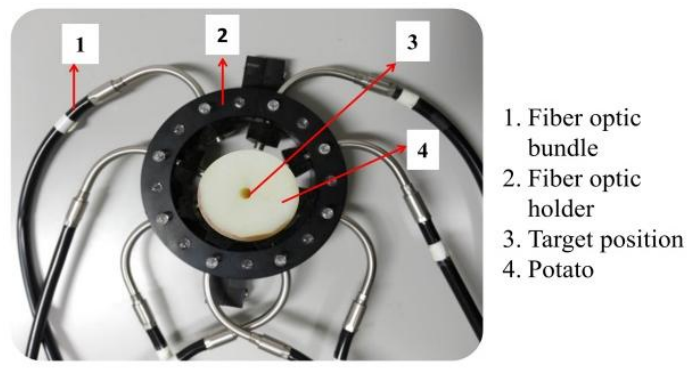

Fig. 4. Experimental configuration

The experimental configuration is shown in Figure 4. In this experiment, the near-infrared laser emits continuous light having peak powers around $0.5 \mathrm{~W}$ at $759 \mathrm{~nm}$. A beam of light is projected to object through a single fiber at the center of fiber optic bundle then the intensity of scattered and absorbed light received by a photodetector via annular fibers within fiber optic bundle.

There are 8 test points, as shown in Figure 4, on the object boundary that will occupied by source and detector. Then, the boundary data matrix is denoted as:

$$
b=\left[\begin{array}{cccc}
b_{1,1} & b_{2,1} & \cdots & b_{8,1} \\
b_{1,2} & b_{2,2} & \cdots & b_{8,2} \\
\vdots & \vdots & \ddots & \vdots \\
b_{1,8} & b_{2,8} & \cdots & b_{8,8}
\end{array}\right]_{N S x N D}
$$

where NS indicate number of sources and ND indicate number of detectors.

\section{RESULTS AND ANALYSIS}

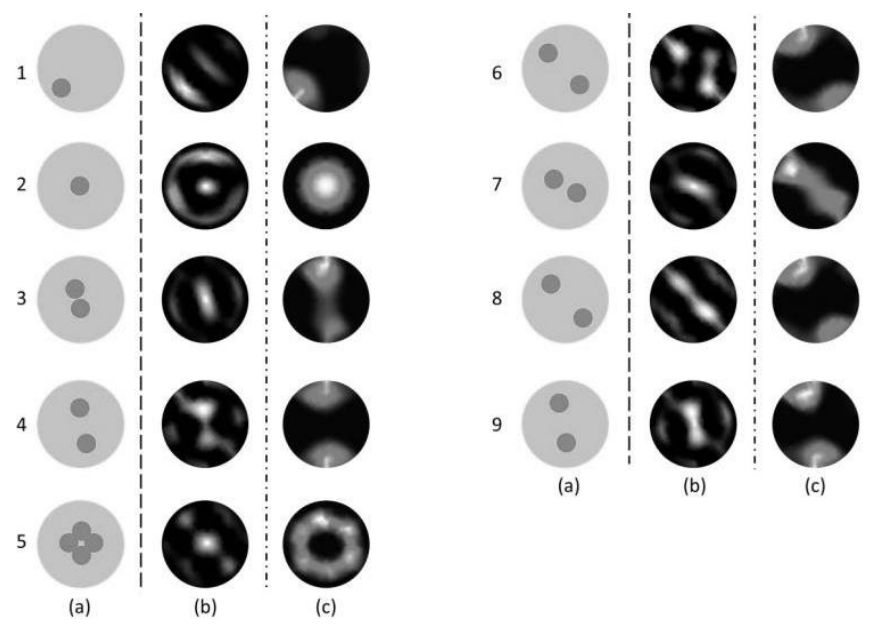

Fig. 5. The results of CW-NIMoBIR reconstruction(a) the depiction of object condition (b) The reconstructed image using RTOT system (c) The reconstructed image using DOT system. 
The experiment objects with various target positions and the reconstructed images are shown in Figure 5. Based on visual analysis, in general, we can conclude that the NIMoBIRDOT algorithmis succeeded to represent the position and size of the objects. However, there are several white spots on reconstructed imagesthat are difficult to distinguish from the presence of the target. Furthermore, this method is not able to show the separation between two targets on object.Meanwhile, as shown in Figure 5,the NIMoBIR TTRO has a better ability to separate two targets on object than the NIMoBIR TDO algorithm.However, this method still has weakness to reconstruct the target position. In order to carry out an objective analysis, the residual value is used to show the difference between measurement and reconstructed boundary data, as shown in Table $\mathbf{1}$.

TABLE I. NUMERICAL ANALYSIS CALCULATION RESULTSON NIMOBIRCWDOT AND RTOT

\begin{tabular}{|c|c|c|}
\hline \multirow{2}{*}{ Object } & \multicolumn{2}{|c|}{ Residual Value } \\
\cline { 2 - 3 } & DOT & RTOT \\
\hline 1 & $8,82 * 10^{-7}$ & $6,5^{*} 10^{-12}$ \\
\hline 2 & $1,2 * 10^{-6}$ & $8,2^{-6} 10^{-9}$ \\
\hline 3 & $1,91 * 10^{-6}$ & $7,49 * 10^{-12}$ \\
\hline 4 & $5,33^{*} 10^{-6}$ & $2,87 * 10^{-12}$ \\
\hline 5 & $4,15^{*} 10^{-6}$ & $3,36^{*} 10^{-13}$ \\
\hline 6 & $2,3 * 10^{-6}$ & $1,08 * 10^{-9}$ \\
\hline 7 & $2,4 * 10^{-6}$ & $6,34 * 10^{-12}$ \\
\hline 8 & $1,93 * 10^{-6}$ & $8,36^{*} 10^{-10}$ \\
\hline 9 & $2,58^{-6} 10^{-6}$ & $3,73 * 10^{-12}$ \\
\hline
\end{tabular}

As shown in Table $\mathbf{1}$, the averages of residual valueon NIMoBIR CW DOT and RTOT are $2.52 * 10^{-6}$ and $1.13 * 10^{-}$ ${ }^{9}$ respectively. These averages are relatively low, so we can conclude that both algorithms are able to reconstruct the boundary data of the real object. The lower residual value obtained by applying NIMoBIR in RTOT system showed that this algorithm has a better performance to represent the measurement boundary data than the use of NIMoBIR in DOT system.Based on the visual and residual analysis, we can conclude that the algorithm NIMoBIR withcontinuous-wave domain in DOT and RTOT systems have the potential to reconstruct the absorption coefficient distribution of agricultural products, such as potato.

\section{CONCLUSIONS}

In this article, we present non-iterative model based image reconstruction for the continuous-wave domain diffusion and radiative transport equations. Experiments using potato with the epoxy targets were conducted to demonstrate our algorithm. The result of this experiment has shown that the NIMoBIR in DOT and RTOT systems have high possibility to detect defects on agricultural produces. However, in the future works, we are going to improve the accuration of reconstructed images.

\section{REFERENCES}

[1] A. Marjono, A. Yano, S. Okawa, F. Gao, and Y. Yamada, "Total light approach of time domain fluorescence diffuse optical tomography", Optics Express, vol. 16, no. 17, pp. 13104-13121, 2008.

[2] A. Zacharopoulos, M. Schweiger, V. Kolehmainen, and S. Arridge, "3D shape based reconstruction of experimental data in Diffuse Optical Tomography", Optics Express, vol. 17, Issue 21, pp. 18940-18956, 2009.

[3] E. Kate K, Henri S. Tapp, Richard B, Robert O. M, and Anthony J.P, "Feasibility study of NIR diffuse optical tomography on agricultural produce", Science Direct Postharvest Biology and Technology, vol. 48, issue 2, pp. 223-230, 2008.

[4] H. Egger, M. Freiberger, and M. Schlottbom, 2010, "On forward and inverse models in fluorescence diffuse optical tomography", Inverse problems and Imaging, vol.4, no. 3, pp. 411-427,

[5] H. Gao and H. Zhao, "A fast forward solver of radiative transfer equation", Transport Theory and Statistical Physics, vol. 38, pp. 149192, 2009.

[6] Harsimrat Singh et al., "Mapping cortical haemodynamics during neonatal seizures using diffuse optical tomography: A case study", NeuroImage: Clinical, vol. 5, pp. 256-265, 2014.

[7] J. Chamorro et al., 2011, "Feasibility of U-curve method to select the regularization parameter for fluorescence diffuse optical tomography in phantom and small animal studies", Optics Express, vol. 19, Issue 12, pp. 11490-11506, 2011.

[8] Lee O., Kim J. M., Bresler Y., and Ye J.C., "Compressive Diffuse Optical Tomography Noniterative exact reconstruction using joint sparsity", IEEE Trans. Med. Imaging, vo. 30, issue 5, pp 1129-1142, 2011.

[9] M. L. Flexman, M. A. Khalil, R. Al Abdi, H. K. Kim, C. J. Fong, E. Desperito, D.L. Hershman, R. L. Barbour, and A. H. Hielscher, "Digital optical tomography system for dynamic breast imaging", J. Biomed Opt, vol. 16 issue 7: 076014, 2011.

[10] M. Panayiotou, A. Gibson, and Prof. J. Hebden, "3Dimensional Optical Tomography of an Unfertilised Chicken Egg”, BSc Thesis, Medical Physics, University College London, 35 p, 2005

[11] P.C. Hansen, "Discrete Inverse Problems Insight and Algorithms", SIAM, 2010.

[12] S. Gupta, Phaneendra K. Y, D. Roy, D. Piao, and Ram M. V., "Singular value decomposition based computationally efficient algorithm for rapid dynamic near-infrared diffuse optical tomography", Medical Physics, vol. 36 , no. 12 , pp. 5559-5567, 2009.

[13] S. Okawa et al.., "Phantom and mouse experiments of time-domain fluorescence tomography using total light approach", Biomed.Opt. Express, vol. 4, no.4, pp. 635-651, 2013.

[14] S. Z.M. Muji et al., "Optical tomography hardware development for solid gas measurement using mixed projection", Flow Measurement and Instrumentation, vol. 33, pp. 110-121, 2013.

[15] Shay Keren, O.Gheysens, C. S. Levin, and S. S. Gambhir, "A comparison between a time domain and continuous wave small animal optical imaging system", IEEE Medical Imaging, vol. 27, no. 1, pp. 5863, 2008.

[16] T. Tarvainen et al., "An approximation error approach for compensating for modelling errors between the radiative transfer equation and the diffusion approximation in diffuse optical tomography", Inverse Problems, vol. 26, 015005, 2010.

[17] V. Nadhira, D. Kurniadi, and E. Juliastuti, "Feasibility Study on Image Reconstruction of Continuous Wave Domain Diffuse Optical Tomography for Quality Control on Seed Potatoes", IEEE, ICICI-BME Conference Proceeding, pp. 421 - 424, 2013.

[18] V. Nadhira, D. Kurniadi, E. Juliastuti, and Adeline Sutiswan, "Study of continuous-wave domain fluorescence diffuse optical tomography for quality control on agricultural produce", AIP Conference Proceedings, vol. 1589, pp. 276-280, 2014.

[19] V. Nadhira, D. Kurniadi, E. Juliastuti, and R. Richo Eka, "Image Reconstruction of Time Domain Diffuse Optical Tomography for Quality Control on Seed Potatoes", IEEE, ICA Conference Proceedings, pp. 266-269, 2013. 
[20] Vebi Nadhira, E. Juliastuti, Agah D. Garnadi and Deddy Kurniadi, "Non Iterative Image Reconstruction Simulation of Continuous Wave Domain Diffuse Optical Tomography for Quality Control on AgriculturalProduce", submitted to International Journal of Modeling, Simulation, and Scientific Computing, 2015.

[21] Xiaofeng Zhang, "Instrumentation in Diffuse Optical Imaging", Photonics, vol. 1, no. 1, pp. 9-32, 2014. 